\title{
Visual Function Questionnaire as an outcome measure for homonymous hemianopia: subscales and supplementary questions, analysis from the VISION trial
}

\author{
Fiona J. Rowe ${ }^{1} \cdot$ Lauren R. Hepworth ${ }^{1}{ }^{1} \cdot$ Elizabeth J. Conroy ${ }^{2} \cdot$ Naomi E. A. Rainford $^{2} \cdot$ Emma Bedson $^{3}$. \\ Avril Drummond ${ }^{4} \cdot$ Marta García-Fiñana ${ }^{2} \cdot$ Claire Howard $^{5} \cdot$ Alex Pollock $^{6} \cdot$ Tracey Shipman $^{7} \cdot$ Caroline Dodridge $^{8}$. \\ Stevie Johnson ${ }^{9} \cdot$ Carmel Noonan $^{10} \cdot$ Catherine Sackley $^{11}$
}

Received: 5 January 2019 / Revised: 27 February 2019 / Accepted: 4 April 2019 / Published online: 17 April 2019

(c) The Royal College of Ophthalmologists 2019

\begin{abstract}
Background: We conduct supplementary analyses of the NEI VFQ-25 data to evaluate where changes occurred within subscales of the NEI VFQ-25 leading to change in the composite scores between the three treatment arms, and evaluate the NEI VFQ-25 with and without the Neuro 10 supplement.

Methods: A prospective, multicentre, parallel, single-blind, three-arm RCT of fourteen UK acute stroke units was conducted. Stroke survivors with homonymous hemianopia were recruited. Interventions included: Fresnel prisms for minimum $2 \mathrm{~h}, 5$ days/week over 6-weeks (Arm a), Visual search training for minimum $30 \mathrm{~min}, 5$ days/week over 6-weeks (Arm b) and standard care-information only (Arm c). Primary and secondary outcomes (including NEI VFQ-25 data) were measured at baseline, 6, 12 and 26 weeks after randomisation.

Results: Eighty seven patients were recruited (69\% male; mean age (SD) equal to 69 (12) years). At 26 weeks, outcomes for 24, 24 and 22 patients, respectively, were compared to baseline. NEI VFQ-25 (with and without Neuro 10) responses improved from baseline to 26 weeks with visual search training compared to Fresnel prisms and standard care. In subscale analysis, the most impacted across all treatment arms was 'driving' whilst the least impacted were 'colour vision' and 'ocular pain'.

Conclusions: Composite scores differed systematically for the NEI VFQ-25 (Neuro 10) versus NEI VFQ-25 at all time points. For subscale scores, descriptive statistics suggest clinically relevant improvement in distance activities and visionspecific dependency subscales for NEI VFQ-25 scores in the visual search treatment arm. Trial Registration: Current Controlled Trials ISRCTN05956042.
\end{abstract}

\section{Background}

Homonymous hemianopia results in loss of one-half of the visual field in both eyes [1,2]. The mean prevalence of visual field loss following stroke has been reported as $31 \%$, although there are large variations in figures reported by individual studies [3].

Homonymous visual field defects can have a severe impact on functional ability and quality of life following stroke [4, 5]. Patients with visual field defects report increased risk of falling, impaired ability to read, altered

Fiona J. Rowe

rowef@liverpool.ac.uk

Extended author information available on the last page of the article activities of daily living, loss of confidence and institutionalisation $[4,6,7]$. There may also be an impact on the patient's ability to participate in rehabilitation as a result of visual field loss which may ultimately affect prognosis and long-term recovery [7]. There is an increased risk of accidents or injuries with visual field loss, which subsequently has cost implications to the NHS and society [8].

Two clinically used interventions to improve vision in hemianopia are visual search compensatory training and provision of monocular prisms [9]. These interventions for homonymous hemianopia were evaluated by a Cochrane systematic review and limited evidence was found in favour of visual search training [10]. Aimola et al., subsequently reported a trial of visual search training for homonymous hemianopia and provided evidence of improved quality of life in the intervention group [11]. Insufficient evidence was 
found by the Cochrane review relating to prisms as an intervention for hemianopia [10].

The National Eye Institute Visual Function Questionnaire-25 (NEI VFQ-25) was designed to measure vision-related quality of life[12]. This outcome tool has been used in several studies to measure quality of life in stroke survivors with visual field loss [13-18]. These studies have all reported subscale scores separately in addition to the composite score having used the NEI VFQ-25 without the additional Neuro 10 supplement (Neuro 10) [19]. This raises the question of whether the Neuro 10 supplement is appropriate for assessing outcome in populations experiencing visual field loss due to neurological aetiology. Neuro 10 was developed with the aim of adapting the NEI VFQ to be better targeted to a neurological population [19]. It is important to select an outcome measure instrument that it is both valid and acceptable for the population of interest, which does not include irrelevant questions and considers the burden of completion [20-22].

The Visual Impairment after Stroke: Intervention Or Not (VISION) pilot trial sought to evaluate visual search training versus prism therapy versus standard care (control) [23]. In particular, the primary objective of VISION was to estimate the parameters required for the calculation of sample size for a definitive trial. Secondary measures included Rivermead Mobility Index, NEI VFQ-25 (Neuro 10), Nottingham Extended Activities of Daily Living, EuroQol, Short Form-12 questionnaires and Radner reading ability. We previously reported that visual function using the NEI VFQ-25, including the Neuro 10 supplement, improved at 26 weeks in the visual search training arm when compared to the Fresnel prisms and standard care arms, with no evidence of differences across arms with other secondary outcomes [24]. At that stage, a detailed analysis of the subscales of the NEI VFQ-25 (Neuro 10) was not conducted. However the data collected from participants within this trial provide a valuable opportunity to explore the subscale analysis and additional information, if any, gained from administering the Neuro 10 supplement in addition to the standard NEI VFQ-25.

The aims of this analysis were to evaluate where changes occurred within subscales of the NEI VFQ-25 leading to change in the composite score between the three treatment arms and to evaluate the NEI VFQ-25 with and without the Neuro 10 supplement.

\section{Methods}

Detailed trial methodology has been published elsewhere $[23,24]$. Briefly, the VISION trial was a randomised controlled, multicentre pilot trial with NHS research ethical approval (10/H1003/119). Participants were recruited from stroke units based in 14 NHS Trusts and randomised to one of three possible treatment arms: prism therapy, visual search training or standard NHS care.

Participants were eligible for inclusion if they met the criteria:

a. 18 years of age or older;

b. Best corrected visual acuity of 0.5 or better in each eye at distance;

c. Stable homonymous hemianopia (partial or complete) induced by recent stroke, defined following WHO guidelines, present over 2 weeks (to exclude rapid recovery cases) but less than 26 weeks prior to randomisation;

d. Refractive error within \pm 5 Dioptres;

e. Willing and able to give consent for the study;

f. Prior to stroke able to read and understand English.

Participants were not eligible for inclusion if they were:

a. unable to consent due to severe cognitive impairment;

b. assessed to have ocular motility impairment and/or visual inattention in addition to the visual field impairment; or

c. had pre-existent visual field impairment due to previous stroke.

Participants eligible for inclusion, and providing consent, attended a baseline assessment, which included assessment and documentation of patient demographics, visual signs and symptoms, visual acuity measures, any additional ocular problems, comorbidity, severity of stroke and level of disability.

This study focuses on the data analysis from the NEI VFQ-25 and supplementary Neuro 10. The NEI-VFQ 25 is composed of 12 subscales, 11 of which are vision-related: general health (1 item), general vision (1 item), near vision activities (3 items), distance vision activities (3 items), social functioning (2 items), role limitation (2 items), dependency (3 items), mental health (4 items), driving (3 items), peripheral vision (1 item), colour vision (1 item) and ocular pain (2 items) [12]. The instrument provides an overall composite score by averaging the 11 vision-related subscales. Both composite and subscale scores range from 0 ("worst functioning") to 100 ("best functioning") [25]. The Neuro 10 is composed of 10 items; tired eyes, bright sunlight, parking a car, using a computer, two eyes seeing differently, eye/lid appearance unusual, blurred vision, trouble focusing on moving objects, binocular double vision and ptosis [19]. While guidelines for the Neuro 10 demonstrate how to merge supplement items with the NEI VFQ-25 to compute an overall score, they do not map onto subscales. The additional Neuro 10 items were included in the existing subscales of the NEI VFQ-25, by consensus using an expert panel, comprising four expert neuroorthoptists (from the British and Irish Orthoptic Society Stroke and Neuro-rehabilitation Clinical Advisory Group). The expert panel achieved immediate consensus on the 
classification of seven of the ten items of the Neuro 10 supplement into the sub scales of the NEI VFQ-25 (Table 1). The remaining three items were discussed by these experts and consensus agreed during a second discussion.

A full statistical analysis plan, which rigorously describes the statistical analysis and methods used, was developed and approved prior to the conduction of this analysis. Descriptive analysis was performed with the use of SAS software, version 9.3 (SAS Institute) and according to the intention-to-treat principle. Scores were calculated on patients with data at both time points only, no imputation methods were used. As the VISION trial was not powered to identify differences, and this analysis is on data collected as a secondary outcome, results should be interpreted with caution and are exploratory only. No formal statistical testing was undertaken.

The analysis of the NEI VFQ-25 (Neuro 10) followed the same principles as in the main analysis of the VISION trial $[12,13]$. To check the robustness resulting from mapping the additional ten items to the standard NEI VFQ-25, analyses were also performed separately on NEI VFQ-25 data as a sensitivity measure. A clinically significant change was defined as 10 points difference [26, 27]. Data from baseline, 26 weeks (final follow up), and the difference between these two time points are presented descriptively overall, and split by treatment arm and subscale. Count data was summarised by counts and percentages. Continuous outcomes are summarised using means and standard deviations since no significant deviations from normality were observed.

\section{Results}

\section{Participants}

Between 17 May 2011 and 9 September 2013, 87 participants were recruited from 1171 stroke survivors assessed for eligibility. The reasons for not being eligible and for refusing to consent were recorded and have been published [28]. The 87 participants were randomised, 27 to Fresnel prisms, 30 to visual search training and 30 to standard care. Two participants $(2.3 \%)$ withdrew from data analysis and follow-up; nine $(10.3 \%)$ from follow-up only and five $(5.7 \%)$ were lost to follow-up, of which four were from the standard arm. At 26 weeks follow-up, there were 24 $(88.9 \%)$ in Fresnel prisms, $25(83.3 \%)$ in visual search training and $22(73.3 \%)$ in standard care. NEI VFQ-25 (Neuro 10) data was available at baseline for 83 participants in total; 25 participants in Fresnel prisms, 30 in visual search training and 28 in standard care. At 26 weeks followup, NEI VFQ-25 (Neuro 10) data was available for 68 participants in total; 24 participants in Fresnel prisms, 25 in visual search training and 19 in standard care.

Participant demographic and clinical characteristics of all randomised participants at baseline are outlined fully in the

Table 1 Classification of additional items of the NEI VFQ-25 (10) supplement on to subscales of the NEI VFQ-25

\begin{tabular}{|c|c|c|c|}
\hline Item & Discussion 1 & Discussion 2 & Subscale agreed \\
\hline $\begin{array}{l}\text { 1. How much difficulty do you have performing tasks when your } \\
\text { eyes are tired? }\end{array}$ & $\begin{array}{l}\text { Consensus not achieved } \\
3 \text { general vision } \\
1 \text { ocular pain }\end{array}$ & Consensus achieved & General vision \\
\hline $\begin{array}{l}\text { 2. Because of your vision, how much difficulty do you have } \\
\text { identifying objects or performing tasks in bright sunlight? }\end{array}$ & Consensus achieved & & General vision \\
\hline $\begin{array}{l}\text { 3. Because of your vision, how much difficulty do you have } \\
\text { parking a car? }\end{array}$ & Consensus achieved & & Driving \\
\hline $\begin{array}{l}\text { 4. Because of your vision, how much difficulty do you have } \\
\text { using a computer? }\end{array}$ & Consensus achieved & & Near activities \\
\hline $\begin{array}{l}\text { 5. I have a feeling that my two eyes see differently, even with } \\
\text { correction (glasses or contact lenses) }\end{array}$ & Consensus achieved & & General vision \\
\hline 6. I have a feeling that my eye or eyelid appearance is unusual & $\begin{array}{l}\text { Consensus not achieved } \\
2 \text { vision specific social } \\
\text { functioning } \\
2 \text { general vision }\end{array}$ & Consensus achieved & $\begin{array}{l}\text { Vision specific social } \\
\text { functioning }\end{array}$ \\
\hline 7. My vision is blurry, not clear, or "fuzzy" & Consensus achieved & & General vision \\
\hline 8. I have trouble focusing on or following moving objects & Consensus achieved & & General vision \\
\hline $\begin{array}{l}\text { 9. I have double vision with both eyes open that is not present } \\
\text { when either eye is covered }\end{array}$ & Consensus achieved & & General vision \\
\hline 10. My eyelid(s) droop & $\begin{array}{l}\text { Consensus not achieved } \\
2 \text { vision specific social } \\
\text { functioning } \\
2 \text { general vision }\end{array}$ & Consensus achieved & General vision \\
\hline
\end{tabular}


main results paper [24]. There were no notable differences at baseline between the three arms for participant demographics. The population consisted primarily of white (97.6\%) males $(69.4 \%)$ with an average age of 69 years, randomised, on average, at 11 weeks post-stroke stroke. The stroke location was mostly classified as unilateral $(43.5 \%$ left; $54.1 \%$ right), with $47(55.3 \%)$ complete and 38 $(44.7 \%)$ partial homonymous hemianopia.

\section{Composite scores}

The mean (SD) composite score of the NEI VFQ-25 with Neuro 10 from all participants was 63.2 (18.3) at baseline and 65.9 (20.5) at 26 weeks follow-up [24]. The mean (SD) composite score of the NEI VFQ-25 without Neuro 10 from all participants was $54.6(17.7)$ at baseline and 56.3 (19.6) at 26 weeks. The mean (SD) difference across the three treatment arms between baseline and 26 week follow-up with Neuro 10 was 2.6 (15.2) and without Neuro 10 was 1.8 (14.0). The composite scores across the three treatment arms for baseline and 26 weeks follow-up with and without the Neuro 10 supplement are outlined in Table 2.

Notable differences were present at baseline between the three arms for NEI VFQ-25 data with higher scores for the Fresnel prism arm versus standard care and visual search training arms. The average composite without Neuro 10 score (SD) for the Fresnel prism arm at baseline was the highest of the three treatment arms at 59.5 (15.5), with the visual search training arm being the lowest at 51.7 (18.8) and standard care being 52.4 (18.3). The average composite with the Neuro 10 were consistently higher across the three treatment arms with Fresnel prims arm at 68.5 (16.2), visual search training at 59.5 (19.0) and standard care being 61.8 (SD 19.2).

The remainder of the analysis refers to the NEI VFQ-25 without the Neuro 10 supplement. The only treatment arm to show improvement in the average composite score (SD) was the visual search training arm with a mean difference of 7.2 (15.5) at 26 weeks follow-up when compared to baseline, resulting in a composite score of 58.9 (19.2) at 26 weeks. The Fresnel prism and standard care arms dropped slightly by -0.9 (13.1) and $-2.1 \quad$ (11.1) respectively.

\section{Subscale scores}

The subscale scores are outlined in Table 3. The most impacted subscale across all treatment arms was driving, with the average score (SD) being 3.5 (15.1) from a maximum score of 14 within this subscale. The least impacted subscale across all three treatment arms was colour vision at 89.8 (17.9), followed by ocular pain at 84.9 (22.1), however the score for the latter dropped in the standard care arm at 26 weeks follow-up by 6.6 (23.8).
The change in scores between baseline and 26 week follow-up is displayed in Fig. 1. Overall, the scores across ten of the twelve subscales improved between baseline and 26 weeks follow-up. The remaining two subscales (general health and colour vision) scores deteriorated. None of the changes for the overall cohort exceeded the clinically significant figure of 10. The Fresnel prism arm improved in four subscales (general vision, ocular pain, near activities and peripheral vision) and deteriorated in seven (general health, distance activities, vision-specific social functioning, vision-specific mental health, vision specific role difficulties, vision-specific dependency, colour vision) subscales. None of the changes for the Fresnel prism arm exceeded the clinically significant figure of 10 . The visual search training arm improved in ten subscales (general vision, ocular pain, near activities, distance activities, vision-specific social functioning, vision-specific mental health, vision-specific role difficulties, vision-specific dependency, driving and peripheral vision) and deteriorated in two subscales (general health and colour vision). The change seen in the distance activities, vision-specific role difficulties and vision-specific dependency subscales exceeded the clinically significant threshold of 10 in the visual search training arm; the change in the other subscales did exceed this threshold. The standard care arm improved in two subscales (near activities and vision-specific role difficulties) and deteriorated in seven (general health, general vision, ocular pain, distance activities, vision-specific mental health, vision-specific dependency and colour vision) subscales. The change seen in the vision-specific role difficulties subscale exceeded the clinically significant threshold of 10 in the standard care arm; the change in the other subscales did exceed this threshold.

Across the three treatment arms there were four instances of an improvement greater than the clinically relevant 10 points. Three were in the visual search training arm and one in the standard care arm, across three subscales; distance activities, vision-specific role difficulties and vision-specific dependency. The largest improvement of 15.2 (31.4) was seen in the vision-specific dependency sub-scale for the visual search training arm. The visual search training arm also showed a large improvement of 10.5 (27.8) in the distance activities subscale. Both the visual search training and standard care arms had improvements of 13.6 (25.5) and -10.4 (23.6), respectively in the vision-specific role difficulties subscale.

\section{Discussion}

In this exploratory analysis of the NEI VFQ-25 (with/ without the Neuro 10 supplement) composite and subscale scores, we found 1) at all time points, the composite scores with the Neuro 10 supplement were consistently higher than 


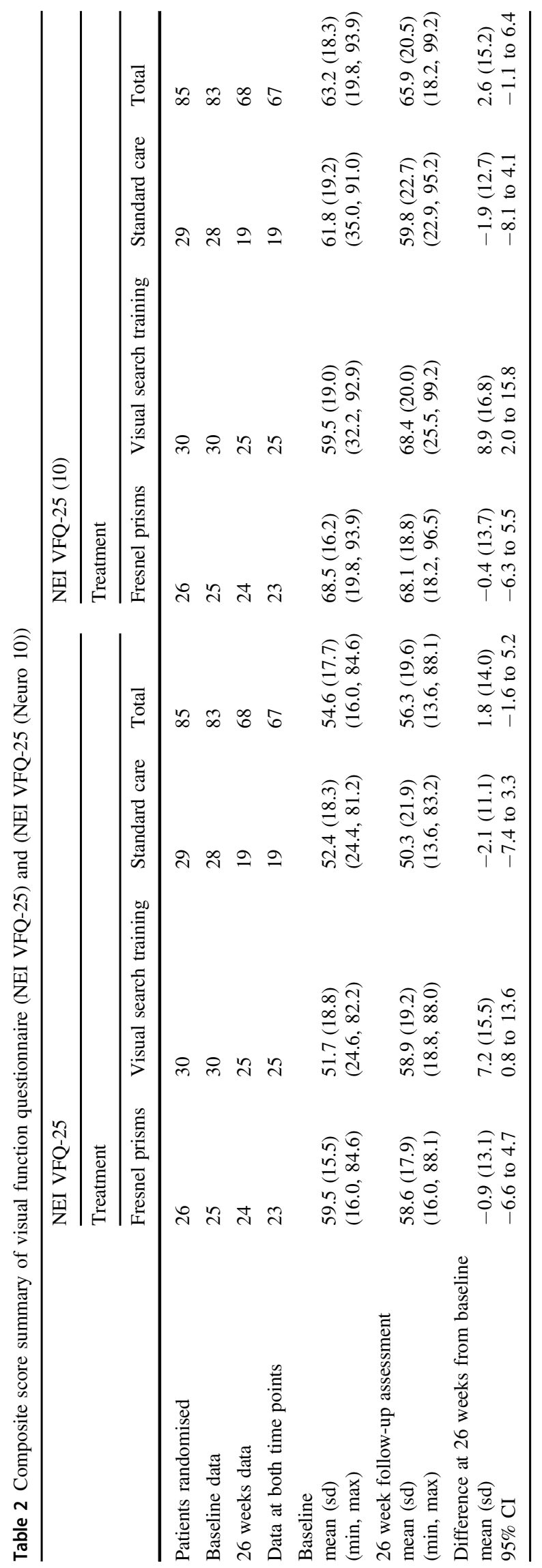

scores for the NEI VFQ-25 without Neuro 10 supplement, and, 2) the subscale changes in each of the treatment arms demonstrated that the visual search intervention had a clinically relevant improvement on distance vision and dependency subscales, but not for other subscales.

The VISION trial asked participants to complete the NEI VFQ-25 with the Neuro 10 supplement; these figures are published alongside other outcome measures elsewhere [24]. The mean composite score when the Neuro 10 supplement was included was systematically higher (63.8 and 65.9) at both baseline and 26 week time points respectively, suggesting consistency in the way it captures aspects of quality of life. A number of the questions included in the Neuro 10 supplement are focused towards ocular motility and central vision problems. The Neuro 10 supplement is recommended for use alongside the NEI VFQ-25 questionnaire in neurological populations. However, the supplementary questions may be suitable for certain populations such as multiple sclerosis where symptoms/signs can also include double vision and eye appearance (reflecting the multiple sclerosis population with which the Neuro 10 supplement was developed [19]). Items such as 'my eye or eyelid appearance is unusual' are not associated with post-stroke hemianopia and therefore responders within this cohort were likely to answer this item 'definitely false' [19]. Scores obtained using the NEI VFQ-25 (Neuro 10) will therefore be higher than scores obtained using the NEI VFQ-25 alone. In addition to the items not being relevant to visual field loss, the inclusion of these additional ten questions for this population potentially results in a higher task burden for the participant and may potentially mask the true impact of the visual field loss. This questions the utility of adding the Neuro 10 supplement to assess vision-related quality of life at specific time points, as well as change in vision-related quality of life over time, when evaluating visual field loss. A future recommendation would be to exclude the Neuro 10 supplement when assessing vision-related quality of life in a population with stroke related visual field loss and using the NEI VFQ-25 only.

Several studies have previously used the NEI VFQ-25 (without Neuro 10 supplement) in stroke populations with homonymous hemianopia. The composite score calculated in this study of 54.6 (SD 17.7) is lower than that reported by other studies. Gall and colleagues reported a composite score of 64.93 (SD 16.01) and 63.98 (SD 16.89) in two studies indicating slightly better quality of life than in the VISION trial. However both studies by Gall et al. did have higher proportions $(58.2 \%$ and $58.4 \%)$ of partial hemianopia/quadrantanopia, i.e. less visual field loss [13, 15]. George and colleagues reported a composite score of 63.6 (SD 18.3) similar to those reported by Gall et al., however their study had a higher proportion (62.5\%) of complete hemianopia [16]. One study by Papageorgiou and colleagues reported the highest composite score of 77.1 which 


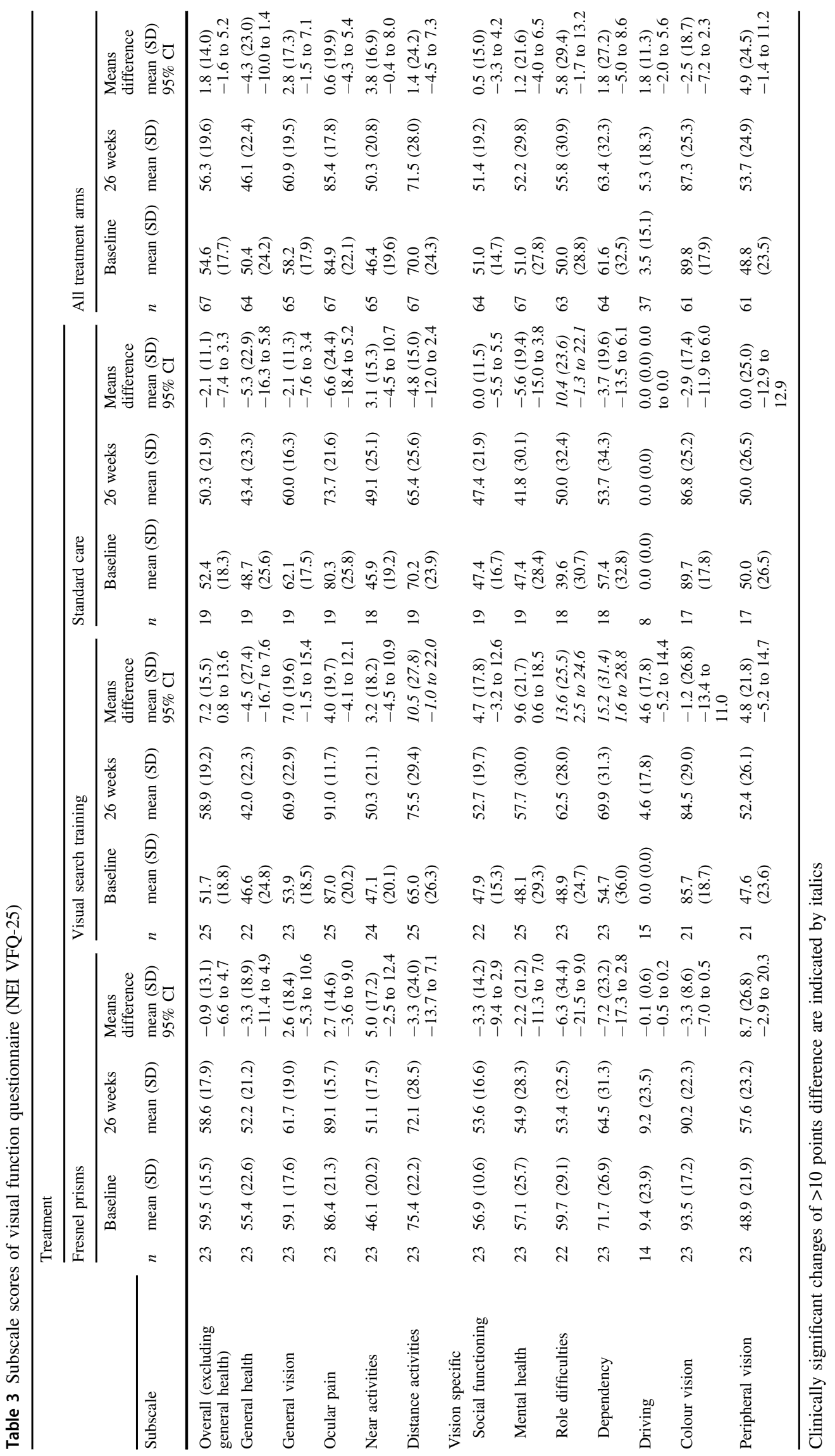


Fig. 1 NEI-VFQ excluding 10item supplement difference in means between baseline and 26 weeks

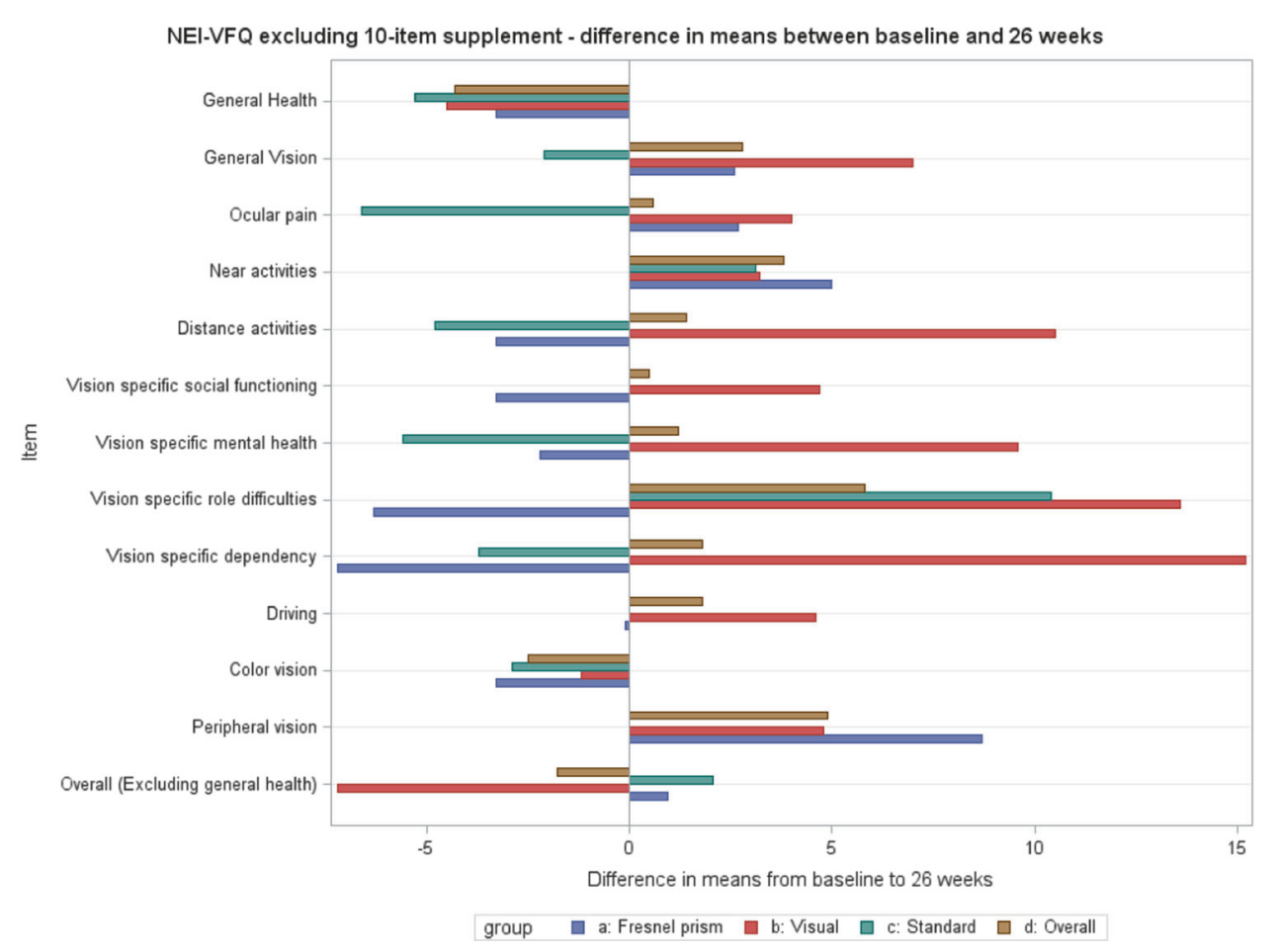

may be the result of less than $33 \%$ of participants having a complete homonymous hemianopia [18].

Gall et al. and Papageorgiou et al. both reported the NEI VFQ-25 subscale scores $[15,18]$. Gall et al. reported nine of the twelve subscales with very similar scores to the findings of this trial [13]. The exceptions to this were near activities at 65.25 (SD 22.69) (18.9 points better than the mean scores in this study); vision-specific social functioning at 74.65 (SD 23.33) (23.7 points better); and driving at 27.35 (SD 33.89) (23.9 points better) [13]. Papageorgiou et al. only reported four of the twelve subscales with similar scores to the findings of this trial. The remaining eight subscales were reported to have consistently better scores, ranging from 14.8 to 31.6 points higher than those found by the current trial [18].

As a cohort, participants were found to improve in all subscales with the exceptions of general health and colour vision, between baseline and 26 weeks. Both of these exceptions were below 10 points which is considered to represent clinical relevance [26, 27]. All subscales saw a minor amount of change between baseline and 26-week follow-up. When split by treatment arm some changes were found to have potential clinical relevance. The distance activities, vision-specific mental health and vision-specific dependency subscales all improved by between a mean of 9.6 to 15.2 in the visual search training arm. The same subscales had slight deterioration in mean score for the Fresnel prism and standard care arms. The vision-specific role difficulties subscale had a mean score improvement of clinical relevance in both the visual search and standard care arms, whereas the Fresnel prism arm had a slight deterioration in mean score. The peripheral vision subscale showed an improvement in mean score for both the Fresnel prism and visual search arms, whereas the standard care mean score remained unchanged between baseline and 26week follow up.

This study is limited as represents a supplementary analysis of a pilot trial that was not powered to identify differences on the NEI VFQ scale. Furthermore, notable differences for NEI VFQ-25 scores at baseline across arms were present (Fresnel prism arm higher than visual search training and standard care). However, results presented are consistent with a larger observational study indicating that these are representative of the wider population with poststroke visual field loss [15]. In addition, unlike other studies, this data was collected as part of a randomised trial in a controlled setting, and therefore adds to the evidence base within the literature and provides scope for further investigation [13, 15, 18]. As such, we would recommend an adequately powered trial is needed to formally compare the differences observed here and to balance for potential differences in scores across treatment arms at baseline and follow-up time points.

\section{Conclusion}

When using the NEI VFQ-25, improvement over time was noted for the visual search training arm specific to distance 
activities and vision-specific dependency subscales only. Scores differed overall for the NEI VFQ-25 (Neuro 10) versus the NEI VFQ-25. The questions contained in the Neuro 10 may not be appropriate to capture aspects of vision that are deficient in patients with hemianopia. We conclude that the NEI VFQ-25 without the Neuro 10 supplement may be more suited for use with populations with stroke-related visual field loss to capture relevant changes of impact on quality of life.

\section{Summary}

\section{What was known before}

- Homonymous visual field defects can have a severe impact on functional ability and quality of life following stroke.

- Patients with visual field defects report increased risk of falling, impaired ability to read, altered activities of daily living, loss of confidence and institutionalisation.

\section{What this study adds}

- Composite scores differed systematically for the NEI VFQ-25 (Neuro 10) versus NEI VFQ-25 at all time points.

- The questions contained in the Neuro 10 may not be appropriate to capture aspects of vision that are deficient in patients with hemianopia. For subscale scores, descriptive statistics suggest clinically relevant improvement in distance activities and vision-specific dependency subscales for NEI VFQ-25 scores in the visual search training arm.

\footnotetext{
Acknowledgements FR had full access to all of the data in the study and takes responsibility for the integrity of the data and the accuracy of the data analysis. We should like to thank the patients and staff who participated in this trial and the following collaborators and investigators: Isabel Ash, Graham Barton, Conrad Beacham, Rachel Breen, Judith Burn, Carol Buckley, Emma Cwiklinski, Joanne Gardiner, Henrietta Holmes-Smith, Sandra Knowles, Tallat Maan, Sonia MacDiarmid, Claire MacIntosh, Lorraine North, Leonie Ripley, Claire Scott, Sarah Spencer, Andrew Twigg, Carole-Anne Vince, Data Monitoring Committee (Cicely Freeman, Irene Stratton, David Wright), Trial Steering Committee (Darren Brand, Catie Bunce, AnneMarie Mackay, Sarah Peel).
}

Funding This trial was funded by the Stroke Association, UK (TSA 2010/02).

Author contributions FR, AD, MGF, SJ, CN, AP and CS conceived of the study, participated in the design and coordination, and helped to draft the manuscript. $\mathrm{LH}, \mathrm{EB}, \mathrm{CD}, \mathrm{CH}$ and $\mathrm{TS}$ participated in the coordination and helped to draft the manuscript. EJC wrote the statistical analysis plan, performed the VFQ 25(10) analysis and supervised the statistical VFQ-25 analysis, participated in the coordination and data monitoring and helped to draft the manuscript. NEAR performed the VFQ-25(10) analysis and helped to draft the manuscript. All authors read and approved the final manuscript.

\section{Compliance with ethical standards}

Conflict of interest The authors declare that they have no conflict of interest.

Ethical approval NHS research ethical approval was given for this trial (10/H1003/119).

Informed consent All participants provided informed, witnessed consent.

Publisher's note: Springer Nature remains neutral with regard to jurisdictional claims in published maps and institutional affiliations.

\section{References}

1. Rowe F, Brand D, Jackson CA, Price A, Walker L, Harrison S, et al. Visual impairment following stroke: do stroke patients require vision assessment? Age Ageing. 2009;38:188-93.

2. Kerkhoff G. Restorative and compensatory therapy approaches in cerebral blindness - a review. Restor Neurol Neurosci. 1999;15:255-71.

3. Hepworth LR, Rowe FJ, Walker MF, Rockliffe J, Noonan C, Howard C et al. Post-stroke visual impairment: a systematic literature review of types and recovery of visual conditions. Ophthal Res. 2016;5. https://doi.org/10.9734/OR/2016/21767

4. Rowe FJ. Stroke survivors' views and experiences on impact of visual impairment. Brain Behav. 2017. https://doi.org/10.1002/ brb3.778

5. Hepworth LR, Rowe FJ. Visual impairment following stroke - the impact on quality of life: a systematic review. Ophthalmol Res. 2016;5:1-15.

6. Ramrattan RS, Wolfs RCW, Panda-Jones S, Jonas JB, Bakker D, Pols HA et al. Prevalence and causes of visual field loss in the elderly and associations with impairment in daily functioning: the Rotterdam Study. Arch Ophthalmol. 2001;119:1788-95.

7. Jones SA, Shinton RA. Improving outcome in stroke patients with visual problems. Age Ageing. 2006;35:560-5.

8. Rowe FJ. Who sees visual impairment following stroke? Strabismus. 2010;18:37-40

9. Hanna KL, Hepworth LR, Rowe FJ. The treatment methods for post-stroke visual impairment: a systematic review. Brain Behav. 2017;7. https://doi.org/10.1002/brb3.682

10. Pollock A, Hazelton C, Henderson CA, Angilley J, Dhillon B, Langhorne P, Livingstone K, Munro FA, Orr H, Rowe FJ, Shahani U. Interventions for visual field defects in patients with stroke. Cochrane Database Syst Rev. 2011;10.

11. Aimola L, Lane AR, Smith DT, Kerkhoff G, Ford GA, Schenk T. Efficacy and feasibility of home-based training for individuals with homonymus visual field defects. Neurorehabilitation Neural Repair. 2014;28:207-18.

12. Mangione CM, Lee PP, Gutierrez PR, Spritzer K, Berry S, Hays RD. Development of the 25-item National Eye Institute Visual Function Questionnaire. Arch Ophthalmol. 2001;119:1050-8. 
13. Gall C, Franke GH, Sabel BA. Vision-related quality of life in first stroke patients with homonymous visual field defects. Health Qual Life Outcomes. 2010;8. https://doi.org/10.1186/1477-7525-8-33.

14. Gall C, Lucklum J, Sabel BA, Franke GH. Vision- and healthrelated quality of life in patients with visual field loss after postchiasmatic lesions. Invest Ophthalmol Vis Sci. 2009;50:2765-76.

15. Gall C, Mueller I, Gudlin J, Lindig A, Schlueter D, Jobke S et al. Vision- and health-related quality of life before and after vision restoration training in cerebrally damaged patients. Restor Neurol Neurosci. 2008;26:341-53.

16. George S, Hayes A, Chen C, Crotty M. Are vision-specific quality of life questionnaires important in assessing rehabilitation for patients with hemianopia post stroke? Top Stroke Rehabil. 2011;18:394-401.

17. Chen CS, Lee AW, Clarke G, Hayes A, George S, Vincent R et al. Vision-related quality of life in patients with complete homonymous hemianopia post stroke. Top Stroke Rehabil. 2009;16:445-53.

18. Papageorgiou E, Hardiess G, Schaeffel F, Wiethoelter H, Karnath $\mathrm{H}-\mathrm{O}$, Mallot $\mathrm{H}$ et al. Assessment of vision-related quality of life in patients with homonymous visual field defects. Graefes Arch Clin Exp Ophthalmol. 2007;245:1749-58.

19. Raphael BA, Galetta KM, Jacobs DA, Markowitz CE, Liu GT, Nano-Schiavi $L$ et al. Validation and test characteristics of a 10item Neuro-Ophthalmic supplement to the NEI-VFQ-25. Am J Ophthalmol. 2006;142:1026-35.

20. Fitzpatrick R, Davey C, Buxton MJ, Jones DR. Evaluating patient-based outcome measures for use in clinical trials. Health Technol Assess. 1998;2. https://doi.org/10.3310/hta2140.

21. Reeve BB, Wyrwich KW, Wu AW, Velikova G, Terwee CB, Snyder $\mathrm{CF}$ et al. ISOQOL recommends minimum standards for patient reported outcome measures used in patient-centred outcomes and comparative effectiveness research. Qual Life Res. 2013;22:1889-1905.

22. de Silva D. Evidence Scan No 18: measuring patient experience The Evidence Centre, The Health Foundation 2013.

23. Rowe FJ, Barton PG, Bedson E, Breen R, Conroy EJ, Cwiklinski $\mathrm{E}$ et al. A randomised controlled trial to compare the clinical and cost-effectiveness of prism glasses, visual search training and standard care in patients with hemianopia following stroke: a protocol. BMJ Open 2014;4. https://doi.org/10.1136/bmjopen2014-005885.

24. Rowe FJ, Conroy EJ, Bedson E, Cwiklinski E, Drummond A, Garcia-Fiñana $\mathrm{M}$ et al. A pilot randomized controlled trial comparing effectiveness of prism glasses, visual search training and standard care in hemianopia. Acta Neurol Scand. 2016;136:310 321.

25. The National Eye Institute 25-Item Visual Function Questionnaire (VFQ-25) Scoring Algorithm. https://www.nei.nih.gov/sites/defa ult/files/nei-pdfs/manual_cm2000.pdf

26. Mangione CM, Berry S, Spritzer K, Janz NK, Klein R, Owsley C et al. Identifying the content area for the 51-item National Eye Institute Visual Function Questionnaire: results for focus groups with visually impaired persons. Arch Ophthalmol. 1998;116:227-33.

27. Mangione CM, Lee PP, Pitts J, Gutierrez P, Berry S, Hays RD. Psychometric properties of the National Eye Institute Visual Function Questionnaire (NEI-VFQ). Arch Ophthalmol. 1998;116:1496-504.

28. Rowe FJ, Conroy EJ, Barton G, Bedson E, Cwiklinski E, Dodridge $\mathrm{C}$, et al. A randomised controlled trial of treatment for post-stroke homonymous hemianopia: screening and recruitment. Neuro-Ophthalmol. 2016;40:1-10.

\section{Affiliations}

Fiona J. Rowe ${ }^{1} \cdot$ Lauren R. Hepworth $\mathbb{1}^{1}$ - Elizabeth J. Conroy ${ }^{2} \cdot$ Naomi E. A. Rainford $^{2} \cdot$ Emma Bedson $^{3}$. Avril Drummond ${ }^{4} \cdot$ Marta García-Fiñana ${ }^{2} \cdot$ Claire Howard $^{5} \cdot$ Alex Pollock $^{6} \cdot$ Tracey Shipman $^{7} \cdot$ Caroline Dodridge $^{8}$. Stevie Johnson ${ }^{9} \cdot$ Carmel Noonan $^{10} \cdot$ Catherine Sackley $^{11}$

1 Department of Health Services Research, University of Liverpool, Liverpool L69 3GB, UK

2 Department of Biostatistics, University of Liverpool, Liverpool L69 3GA, UK

3 Clinical Trials Research Unit, University of Liverpool, Liverpool L69 3GL, UK

4 School of Health Sciences, University of Nottingham, Nottingham NG7 2UH, UK

5 Department of Orthoptics, Salford Royal NHS Foundation Trust, Manchester M6 8HD, UK

6 Nursing, Midwifery and Allied Health Professions Research Unit,
Glasgow Caledonian University, Glasgow G4 0BA, UK

7 Department of Orthoptics, Sheffield Teaching Hospitals NHS Foundation Trust, Sheffield S10 2JF, UK

8 Department of Orthoptics, Oxford University Hospitals NHS Trust, Oxford OX3 9DU, UK

9 Eye Clinic Support Service, Royal National Institute of Blind People, Birmingham B29 6NA, UK

10 Department of Ophthalmology, Aintree University Hospital NHS Foundation Trust, Liverpool L9 7AL, UK

11 Division of Health and Social Care, King's College, London WC2B 5RL, UK 